\title{
IDEAS SOBRE EL CONCEPTO BIOLÓGICO DE POBLACIÓN
}

\author{
BERZAL DE PEDRAZZINI, M. y BARBERÁ, 0 . \\ Departament de Didàctica de les Ciències. Universitat de València. Apartado de Correos 22045, \\ E-46071 Valencia.
}

\section{SUMMARY}

The population concept is one of the foremost in biology, and its comprehension is essential to grasp the genetical and evolutionary aspects of living beings. Nevertheless, school science hardly takes into account the richness and complexity of the biological concept of population. We have carried out this study with the intention of finding out students' ideas about the population concept (and other concepts related to it such as the species concept) and putting forward some hypotheses to explain the origins of these ideas.

\section{INTRODUCCIÓN}

El estudio de las poblaciones de organismos vivos constituye un aspecto central de la biología, y su comprensión es fundamental tanto desde una perspectiva evolutiva como en su dimensión genética. Sin embargo, la ciencia escolar raramente toma en cuenta la riqueza y complejidad de este concepto: «En las situaciones didácticas, hablar de población es generalmente indicar la idea de un grupo de individuos de la misma especie, sin que las implicaciones funcionales y evolutivas del concepto aparezcan» (Develay y Ginsburger-VogeI 1986, p.19). En el contexto social en que se mueven los alumnos el vocablo población es de uso corriente. Todos saben «de qué se trata» cuando se alude a él en un medio en el que esta palabra se asocia con una característica observable de los seres humanos: su condición de organismos sociales. Para Hawley (1975) "considerar cualquier fenómeno social como un agregado de unidades biológicas es el medio más abstracto de verlo, por ello la población constituye el denominador común de todas las Ciencias Sociales».

Existe una serie de problemas asociados con el proceso de enserianza-aprendizaje del concepto de población y su campo conceptual, y su estudio puede partir del conocimiento de las ideas que los alumnos de los distintos niveles de escolaridad obligatoria poseen sobre este concepto y sobre otros estrechamente relacionados con él. A pesar de su importancia, únicamente hemos encontrado en la literatura dos trabajos que tratan de alguna manera este propósito (Ola Adeniyi 1985, Develay y Ginsburger-Vogel 1986), y en ambos se ponen de manifiesto algunas de las dificultades que los alumnos nigerianos y franceses tienen cuando deben manejar el concepto de población.

Estas dificultades pueden suponer especialmente un grave problema para los programas educativos que, como el de Argentina, se basan en un enfoque predominantemente ecologico para la instrucción de los alumnos en biología (DINEMS 1978 y 1980), y ésta es la razón principal por la que hemos realizado el trabajo que a continuación presentamos, cuyos objetivos son:

- La identificación de las concepciones de los estudiantes en relación con el concepto biológico de población, así como otros conceptos significativos dentro de su campo conceptual.

- La formulación de hipótesis orientadoras acerca de las probables causas de las concepciones altemativas halladas.

\section{DISEÑO DE LA INVESTIGACIÓN}

Para la detección de las concepciones de los alumnos se ha confeccionado un cuestionario escrito de base semiestructurada, atendiendo a las ventajas que of rece por las características del trabajo y la población seleccionada para el estudio. El proceso seguido para su diseño se basa en los criterios propuestos por Treagust (1988). En la primera fase de la elaboración del cuestionario se realizó 
Figura 1

Mapa del campo conceptual de población, adaptado a las características del currículo vigente en Argentina.

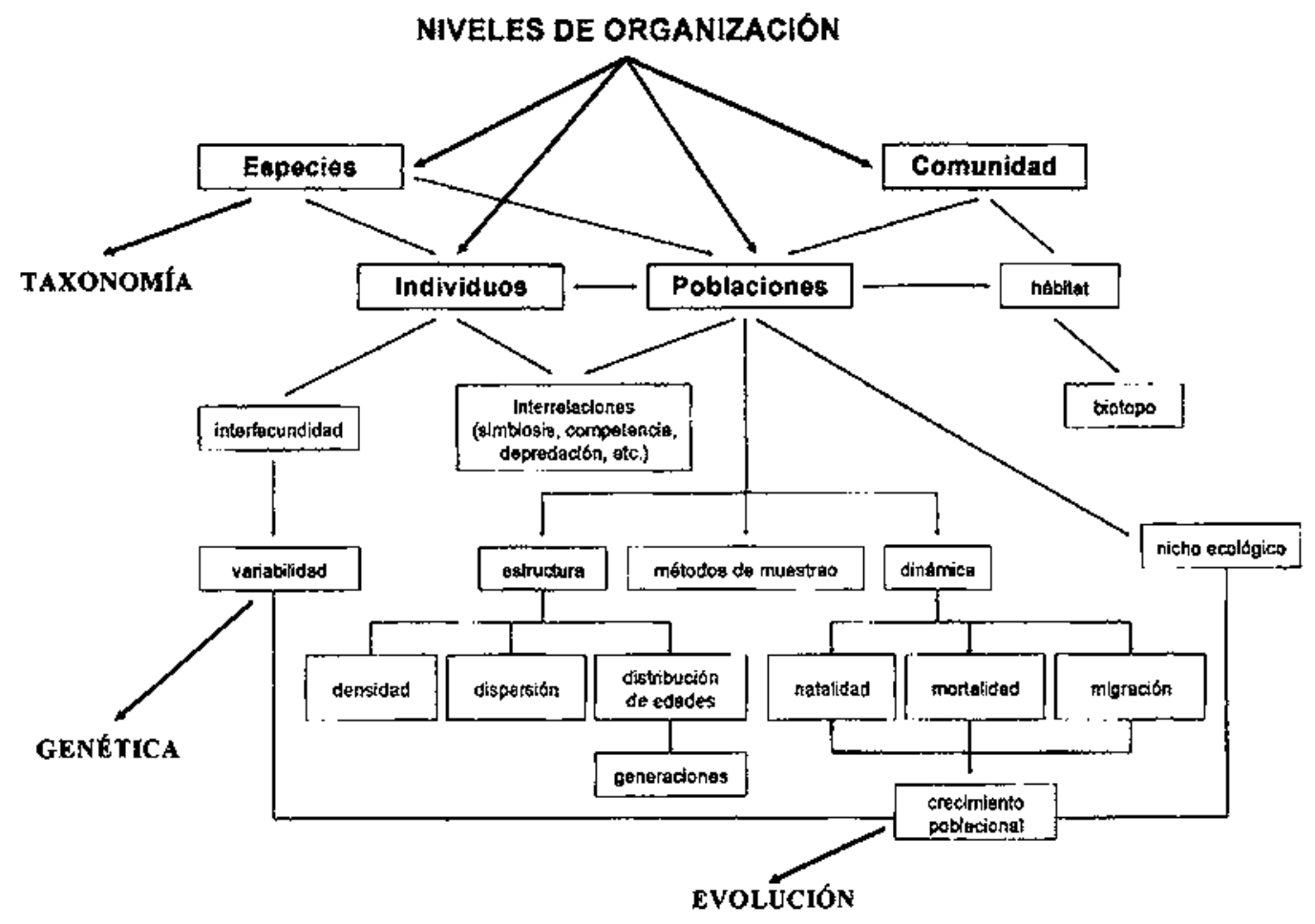

la delimitación y el análisis del contenido sobre el que se pretende inđagar. A tal efecto se construyó un mapa conceptual (Novak y Gowin 1988), tomando como fundamento los Contenidos Mínimos de Ciencias Biologicas aprobados en 1978 por el Consejo Federal de Educación de la República Argentina para el primer curso (13-14 años) del Ciclo Básico Común del Nivel Medio de Educación (Fig. 1). Una versión inicial de preguntas elaboradas se consultaron en un sondeo informal con alumnos de distintas edades y nivel escolar y con adultos no especializados en el tema. El instrumento fue sometido posteriormente a la consideración de una especialista en lengua castellana, a fin de detectar las posibles dificultades procedentes del tipo de lenguaje empleado.

El cuestionario resultante fue aplicado experimentalmente a un grupo de 34 alumnos del primer curso del ciclo básico del nivel medio, a fin de apreciar su accesibilidad en relación con el contexto en que debía emplearse y corregir giros idiomáticos o expresiones que ofrecieran excesiva dificultad. La versión final es la que se describe en este trabajo.

\section{Características del cuestionario}

Comprende cinco secciones en las que se han incluido cuestiones directas que solicitan la definición de los conceptos de población y otros que integran su campo conceptual (Sección I), asî como cuestiones abiertas y cerradas que se estructuran alrededor de un tema o situación, más o menos real, en los que no aparecen explícitos los aspectos a investigar, pero su conocimiento es requerido para of recer explicaciones o soluciones (Secciones II-V).

\section{Características de la población consultada}

Se incluyen en el estudio tres niveles educativos y un total de nueve grupos de alumnos de escuelas de la provincia de Santa Cruz (Argentina), distribuidos según se presenta en el cuadro I.

\section{Cuadro I}

Distribución por niveles de escolaridad, curso y sexo de la población consultada.

\begin{tabular}{|c|c|c|c|c|c|}
\hline $\begin{array}{c}\text { Nivel } \\
\text { educativo }\end{array}$ & $\begin{array}{c}\text { Curso } \\
\text { (grupos) }\end{array}$ & $\begin{array}{c}\text { Edades } \\
\text { (anos) }\end{array}$ & Mujeres & Hombres & TOTAL \\
\hline Primario & $7^{2}(2)$ & $12+13$ & 29 & 21 & 50 \\
\hline Secundario & $1^{n}(3)$ & $13-14$ & 86 & 23 & 109 \\
\hline & $3^{\circ}(2)$ & $15-16$ & 34 & 18 & 52 \\
\hline Superior & $2^{2}(1)$ & $>18$ & 18 & 1 & 19 \\
\hline
\end{tabular}


Gráfico 1

Ideas sobre los conceptos de individuo y población.
Gráfico 2

Ideas sobre el concepto de especie.

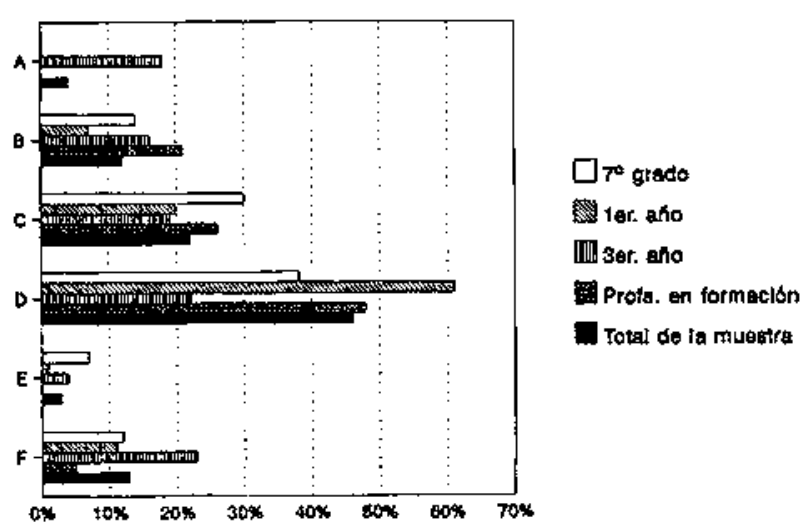

A. Respuestas que introducen en la definición, además de lo especificado en B y C, las variables espacio o tiempo (p.e. el individuo es un ser único y diferente, y la población es un grupo de individuos de la misma especie que se relacionan entre st y comparten el mismo habitat).

B. Respuestas que consideran el concepto de especie (p.e.poblacion es un conjunto de seres de una misma especie).

C. Respuestas que reconocen a la población como un conjunto de individuos, sin mencionar el concepto de especie (p.e. el individuo es uno sólo y la población son muchos).

D. Respuestas que se refieren exclusivamente a los seres humanos y a ja población humana (p.e. el individuo es unapersona y la población es un conjunto de personas).

E. No contestan.

F. Respuestas que confunden el concepto de poblactón con el de comunidad biótica u otros, o que son confusas, contradictorias 0 incomprensibles (p.e. grupo de varias especies o donde viven los individuos).

El cuestionario fue aplicado en la segunda etapa del año lectivo 1990 y los alumnos no habían estudiado el tema Población durante ese curso. Los de $7^{\circ}$ grado de la escuela primaria lo habían tratado en $6^{\circ}$ grado, al igual que en ler. año del cíclo básico del nivel medio. Estos últimos habían estudiado otros conceptos ecológicos durante el período lectivo considerado, pero no abordaron particularmente el concepto de población por encontrarse al final del la planificación prevista. El nivel superior está representado en la muestra por los alumnos de la asignatura Ciencias Biológicas y su Didáctica, correspondiente al segundo curso del profesorado para la educación primaria. Los estudiantes de tercer curso del nivel medio y los del profesorado de educación primaria habían estudiado el tema en dos oportunidades: en $6^{\circ}$ grado del nivel primario y en ler. año del nivel medio.

Los cuestionarios fueron cumplimentados en una clase habitual de ciencias en presencia del profesor, no fijándose límite de tiempo (en general se empleó un módulo de ochenta minutos) y realizándose aclaraciones sólo sobre planteamientos generales del instrumento.

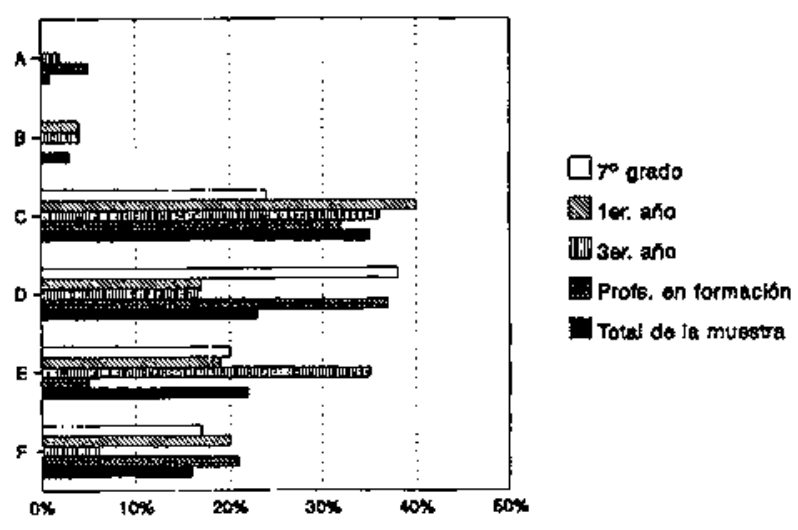

A. Respuestas que incluyen el concepto biologico de especie (p.e. conjunto de individuos que tienen en común suorigen, poseen forma fisica semejante, las mismas costumbres, etc., éstos se reúnen en un lugar geografico, conviven juntos formando una población).

B. Respuestas que consideran el concepto de interfecundidad (p.e. las especies son individuos semejontes y que se reproducen entre s! o ilenen el mismo origen).

C. Respuestas que definen especie por las semejanzas morfológicas entre los individuos o evidencian un concepto tipológico de especie (p.e. la especie es un grupo de individuos con características en común, un tipo de ser vivo, una clase de animal o planta).

D. Respuestas que consiciesan la especie como «razan de seres humanos o exclusivamente de animales (p.e. la especie es la raza del individuo, es la raza de las personas, clase de personas).

E. No contestan.

F. Respuestas confusas, incoherentes o incomprensibles (p.e. se refiere a la especie del individuo que forma la poblacion).

Las respuestas obtenidas fueron interpretadas de manera comparativa estableciéndose categorías; estas categorías no fueron establecidas a priori, sino, como en otros trabajos (Jiménez Aleixandre 1989), después de concre" tar el análisis de las respuestas obtenidas, realizado en tres niveles de aproximación. El primero fue practicado sobre las respuestas dadas a la Sección I, considerando los conceptos de individuo, población y especie. Los datos obtenidos en relación con estos conceptos se agruparon en clusters (Sneath y Sokal 1973), a fin de establecer patrones de respuestas posibles dentro de la población consultada, que fueron orientadores para proponer niveles de conceptualización y redefínir categorías en una segunda etapa. En el tercer nivel y sobre la base de los niveles de análisis ya establecidos, se evaluaron las respuestas obtenidas en las restantes cuestiones, proce * diéndose a la diferenciación en categorías de las respuestas a cada una de las preguntas realizadas.

El número de categorías de respuestas para las distintas cuestiones es en general de seis, con el objeto de cubrir todas las respuestas en forma exhaustiva. Estas categorias son excluyentes entre sí en cuanto a que una respues- 
ta asignada a una de ellas no lo puede ser al mismo tiempo a otra.

Las categorías utilizadas han sido ordenadas en forma decreciente desde el nivel A que refleja el planteamiento conceptual más próximo a la ciencia escolar hasta el nivel $\mathrm{C}$, en un continuo en relación con el conocimiento científico del concepto; el resto de categorías son los niveles D (planteamientos predominantemente antropocéntricos), $\mathrm{E}$ (no contesta) y $\mathrm{F}$ (respuestas incomprensibles, confusas o contradictorias). Mayores precisiones en cuanto a cada categoría se realizan al analizar los resultados obtenidos (Gráficos 1-9).

\section{RESULTADOS}

Los resultados obtenidos se exponen discriminados según las diferentes secciones del instrumento utilizado. Se aborda inicialmente la presentación y comentario de cada pregunta, realizándose posteriormente la discusión de los resultados.

\section{SECCIÓN I}

Explica Las diferencias que existen entre los siguientes pares de paiabras:

1.1. Individuo y población.

1.2. Población y especie.

\section{Objetivos}

Estas cuestiones intentan averiguar cuál es el conocimiento declarativo del concepto de población que utilizan los estudiantes y la relación que mantienen entre este concepto y otros básicos que definen su campo conceptual. Los resultados obtenidos en los distintos grupos consultados se exponen en los gráficos 1 y 2 . En el tratamiento y presentación de los datos del gráfico I se ha optado por mantener relacionados Ios conceptos de individuo y población para una mejor caracterizacióne interpretación de las ideas de los altumnos. Los porcentajes correspondientes a Ias categorías de concepciones sobre especie biológica se pueden contemplar en el gráfico 2 .

\section{Comentarios}

Los datos obtenidos (Gráfico 1) permiten constatar un alto porcentaje de concepciones antropocéntricas (categoría D) en todos los grupos, lo que corresponde a una característica general en el pensamiento de las personas: la tendencia a atribuir a la realidad desconocida las propiedades de los modelos conocidos o más accesibles, adjudicando propiedades animistas al mundo inanimado o explicando la conducta de los organismos con criterios antropocéntricos. La categoría C es la que sigue a la D en número de respuestas, y la interpretamos como el fruto de un pensamiento concreto que se manifiesta en un tipo de generalización como la siguiente: El individuo es uno sólo y la población son muchos. Serrano y Blanco (1988),
Driver y otros (1989) y Pozo y otros (1991) han destacado acerca de las concepciones espontáneas de los alumnos que suelen centrarse casi exclusivamente en Io observable, y esta característica de las poblaciones (Ia abundancia, es decir la cantidad de individuos) es reconocible de manera directa.

Las respuestas correspondientes a la categoría $\mathbf{B}$, que toman en consideración la noción de especie al proponer la definición de población, muestran un ordenamiento sugerente: Ios alumnos de $7^{\circ}$ grado ponen de manifiesto un porcentaje ligeramente superior a los de ler. año, y similar al de los de $3 e r$, año; con muy escasa diferencia, los valores mayores corresponden al grupo de los maestros en formación. Ello indica una escasa incidencia del sistema educativo en la elaboración de la noción de especie, la que se considera como demasiado implícita en la enseñanza ( $Y$ us Ramos 1989). En la muestra total, sólo el 4\% de alumnos elabora una defínición de población que incluya las variables espacio y tiempo, por cuanto la categoría A sólo está presente en el grupo correspondiente al 3er. curso, con un porcentaje bajo de respuestas $(17 \%)$.

Los alumnos que no contestan son pocos (4\% de la muestra). Entre los errores conceptuales detectados debe mencionarse la confusion entre población y comunidad biótica, que se manifiesta con mayor frecuencia en los estudiantes de $3 \mathrm{er}$. año, debido probablemente a que es en este grupo donde se tiene en cuenta precisamente la pertenencia de los individuos a una especie determinada. Algunos alumnos confunden el concepto de población con el de hábitat, lo que interpretamos como otra manifestación de las concepciones antropocéntricas ya citadas.

En general no se observan diferencias importantes entre los grupos pertenecientes a los diferentes niveles educativos.

Las datos expresados en el gráfico 2 ponen de manifiesto las ideas antropocéntricas (D) predominantes en el nivel primario y en los profesores en formación, así como respuestas asociadas con un concepto tipológico de especie o aquellas que se basan en criterios de identificación referidos exclusivamente a los caracteres morfológicos externos (C), concepciones que son mayoritarias en la población consultada. Para Develay y GinsburgerVogel (1986) este tipo de respuesta obedece también at antropocentrismo subyacente. De toda la población, sólo el $3 \%$ hace referencia a la interfecundidad como rasgo distintivo de una especie y únicamente el $1 \%$ sustenta concepciones semejantes al concepto «biologico» de especie. El porcentaje que no contesta (E) en toda la muestra es del $22 \%$, motivo por el que resultan llamativos los altos valores correspondientes al $3 \mathrm{er}$. curso del nivel medio (35\%), mientras que este mismo grupo de alumnos presenta los valores más bajos $(6 \%)$ en la categoría $F$ (respuestas confusas, incoherentes o incomprensibles).

\section{SECCIÓN II}

Linneo, un gran naturalista deI siglo XVIII, calculó que si una planta anual produce tan sólo dos semillas y las 
Gráfico 3

Ideas acerca de la capacidad de multiplicación en las poblaciones.

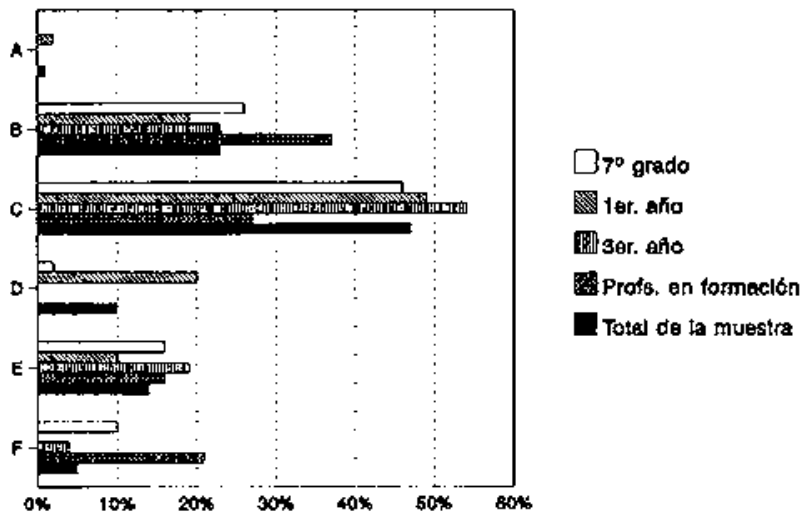

A. Respuestas que hacen referencia a la capaciơad máxima de multiplicación de las poblaciones (p.e. la capacidad de producción de semillas que tienen las plantas y el tiempo que tardan en dar las semillas).

B. Respuestas que consideran la reproducción de los organismos (p.e. la posibilidad de reproducción de los seres vivos o la reproducción de las plantas)

C. Respuestas que reproducen literaimente el enunciado del texto.

D. Respuestas simplistas (p.e. las plantizas y las semillitas y cómo (recen).

E. No contestan.

F. Respuestas confusas, incoherentes o incomprensibles (p.e. el lugar donde se desarrollan las semillas).

plantitas salidas de ellas producen al año siguiente dos, y así sucesivamente, a los treinta años habría millones de plantas desdendientes de la planta original ... (Citado por C. Darwin en El Origen de las Especies).

Contesta las siguientes preguntas:

\section{1. ¿Qué tuvo en cuenta Linneo para efectuar el cálculo?}

2.2. ¿Cómo crees que debería ser la curva que represente mejor los cálculos de Linneo?

2.3. ¿Qué factores bióticos y abióticos actuarían en condiciones naturales, impidiendo el aumento indefinido del número de descendientes?

\section{Objetivos}

Las proposiciones de la teoría de Darwin sobre la evolución por selección natural hacen referencia, entre otras, a las siguientes características de las poblaciones: «Todas las poblaciones poseen la potencialidad de poblar toda la tierra, y lo harían si todos los individuos sobrevivieran y si cada individuo produjera el número máximo de descendientes. Pero no lo hacen: muchos individuos mueren antes de reproducirse, y la mayoría de ellos (si no todos) se reproducen con una tasa inferior a la máxima (...). El número de descendientes que deja un individuo depen-

\section{Gráfico 4}

Construcción de un gráfico representativo de la capacidad de multiplicación en las poblaciones.

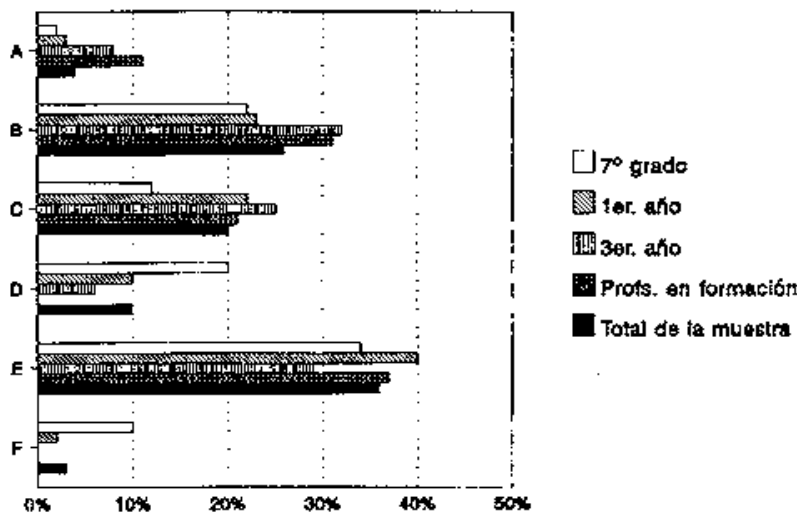

A. Representan una función exponencial o malthusiana.

B. Representan una función lineal.

C. Representan el incremento de la población de manera no convencional.

D. Verbalizan la respuesta, sin representar un grâfico.

E. No contestan.

F. Respuestas confusas, incoherentes o incomprensibles.

de, no por completo pero si de un modo crucial, de la irteracción entre las características y el međio ambiente del mismo» (Begon et al. 1988, pp. 5 y 6). La capacidad máxima de multiplicación poblacional se designa a menudo con la expresión general de potencial bítico o potencial reproductivo. La diferencia entre el potencial biológico máximo y el índice de aumento que se produce en las condiciones reales de laboratorio o de campo se toma a menudo como medida de la resistencia ambiental, que es la suma total de los factores limitativos del medio que impiden que se realice el potencial biótico (Odum 1972).

En las preguntas 2.1 y 2.2 se procura indagar las concepciones de los estudiantes acerca de la noción de potencial reprođuctivo de las poblaciones, así como la habilidad para representar el crecimiento de una población determinada en un sistema de ejes cartesianos. La pregunta 2.3 se formula a fin de constatar los conocimientos que poseen en relación con los factores que actúan regulando el crecimiento poblacional. Es de interés detectar (a través de los ejemplos que proponen) si le asignan importancia a factores denso-independientes o densodependientes de manera más o menos prioritaria, así como el grado de relevancia del factor o factores que mencionan. Los datos obtenidos se presentan en los gráficos 3,4 y 5 .

\section{Comentarios}

Un porcentaje importante de los alumnos de Ios niveles primario y medio de educación contestan a la pregunta 
Grafico 5

Ideas acerca de los factores bióticos y abíticos que impiden el aumento indefinido del número de descendientes.

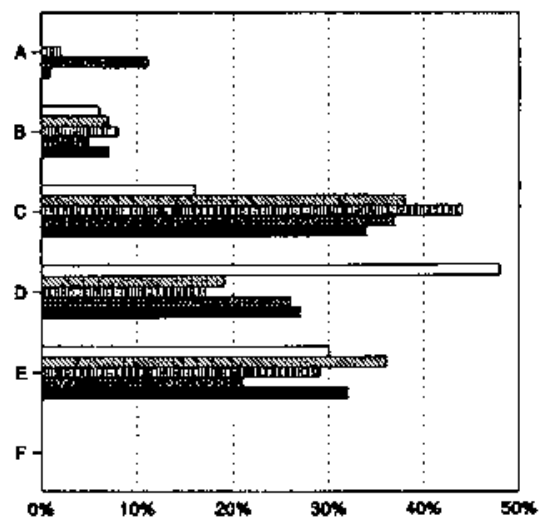

A. Respuestas que hacen referencia a factores dependientes de ta densidad (competencia intraespecífica u otros) e independientes de la densidad (clima u otros) (p.e. factores bióticos comootras plantos que le tapan la luz o depredadores que se comen las plantas, y factores abibticos como la luz, la temperatura, la cantidad de agua y el suelo).

B. Respuestas que mencionan factores biøticos o abioticos relevantes en relación con el problersa que se plantea (p.e. insectos que comen la planta, la lluvia y el tipo de tierra).

C. Respuestas que mencionan factores bioticos o abióticos comparativamente menos relevantes para el problema que se plantea (p.e. el viento, la estación del año).

D. Respuestas que incluyen explicaciones simplistas, ecologistas o antropocéntricas (p.e. los leones, las piedras, el hombre que corta las plantas, que no cuida o no quiere las plantas).

E. No contestan.

F. Respuestas confusas, incoherentes o incomprensibles (no se ha registrado ninguna respuesta en esta categorfa).

\section{Gráfico 6}

Ideas acerca del concepto de densidad de una poblacion.

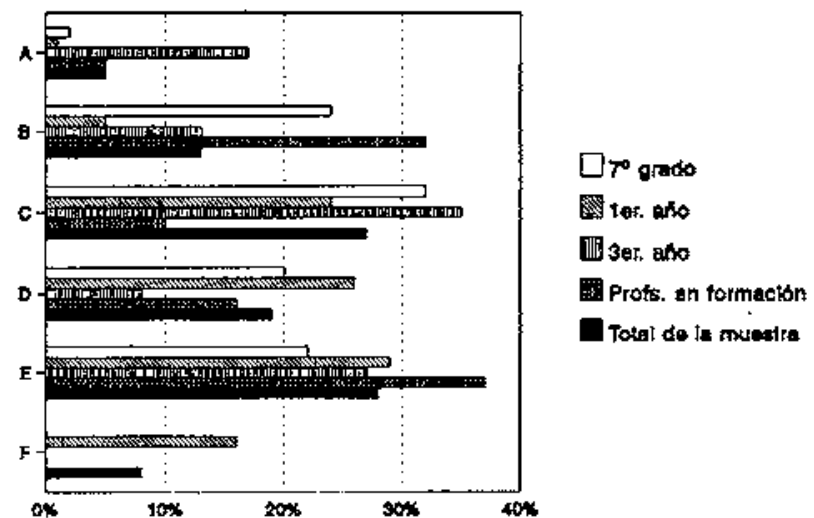

A. Respuestas que indican formas de muestreo y expresan la densidad correctamente, utitizando unidades estándar de medida (p.e. para calcular la densidad de nidos se calculan, por ejemplo, la cantidad de nidos que hay dentro de 3 o 4 zonas de una hectárea, se saca el promedio y se calcula el total aproximado segun la cantidad de hectareas totales del terreno).

B. Respuestas que expresan correctamente (p.e. podemos calcular la densidad contando cuantos nidos hay por $\mathrm{km}^{2}$ ).

C. Respuestas que hacen referencia a algún método indirecto de cálculo, a partir del número (abundancis) de individuos (p.e. calculo la cantidad de nidos a partir del número de parejas que llegan).

D. Respuestas que indican que la densidad se calcuta contando los nioos, con lo que aparece implícito el concepto de abundancia (p.e. contando los nidos).

E. No contestan.

F. Respuestas confusas, incoherentes o incomprensibles (p.e. teniendo en cuenta la situacion climatologica del momento). formulada con una reproducción casi literal del texto (C), lo que se podría interpretar como una deficiencia en el estilo de formulación de la cuestión, o como una evidencia del tipo de mecanización a la que suelen conducir algunas prácticas de lectura comprensiva de textos científicos que se realizan en las escuelas, y que se traducen posteriormente en una lectura superficial y una respuesta acrítica a las cuestiones que se plantean. Los que toman en consideración el concepto de reproducción (B) en relación con la situación planteada representan el 23\% de la muestra total, destacándose el porcentaje obtenido por los profesores en formación (37\%). Unicamente el $1 \%$ de la muestra alude de manera explícita a la capacidad de multiplicación de las poblaciones (gráfico 3).

La representación gráfica que utilizan preferenteremente ( $26 \%$ de la muestra) es la que considera el crecimiento de la población como una función lineal. En general se puede apreciar que, aun careciendo de las destrezas adecuadas para realizar lo solicitado, un grupo de alumnos de ler. año ha intentado superar esta dificultad a fin de poner en evidencia el incremento de la población (categoría C). Es destacable que hay un porcentaje importante de alumnos que no contesta a esta cuestión en todos los cursos y niveles educativos, lo que pone en evidencia la dificultad que entraña para ellos la elaboración de gráficos y otras técnicas cuantitativas (gráfico 4).

Como en otras cuestiones, en la que se refiere a los factores bióticos y abióticos (gráfico 5), también la categoría $\mathrm{C}$, que alude a factores menos relevantes en relación con el problema que se plantea, es la más representada en las respuestas. Destacamos la presencia de explicaciones ecologistas (F) en los alumnos de menor edad en un porcentaje relativamente importante ( $48 \%$ en $7^{\circ}$ grado). Los alumnos que no contestan alcanzan también valores comparativamente altos en esta cuestión (32\% en el total de la población). Esta pregunta permite constatar la dificultad que entraña para los alumnos predecir las múltiples relaciones dentro de los ecosistemas, así como la preponderancia de interpretaciones de cau- 


\section{Gráfico 7}

Ideas acerca de los factores que determinan el crecimiento poblacional.

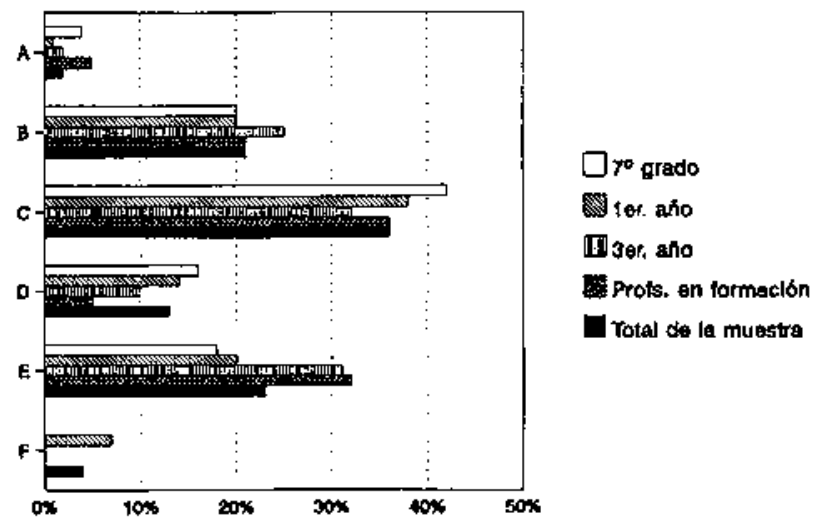

A. Respuestas que establecen relaciones que explican el crecimiento y las fluctuaciones poblacionales (p.e. los factores que intervienen son: el clima, la existencia de un terreno adecuado para los nidos. desocupado por el hombre, los depredadores naturales y la super. vivencia de la mayor cantidad de huevos empollodos, los pingüinos que migran cada año).

B. Respuestas que aluden directa o indirectamente a factores intrínsecos relativos a la dinámica de las poblaciones o factores extrínsecos (p.e. intervienten los factores climaticos, la presencia de gaviotas que se comen los huevos y la cantidad de huevos que ponen).

C. Respuestas que mencionan factores bioticos y abioticos en general o de escasa relevancia para la población (p.e. para producir un aumento en la densidad son los factores abioticos, y para que disminuyan, los bióticos, un verano bueno o malo, las cr(as).

D. Respuestas que proporcionan explicaciones simplistas o ecologistas (p.e. cuando no padecen ni los cazan, que queramos más la naturaleza, to contaminando con petroleo las aguas).

E. No contestan.

F. Respuestas confusas, incoherentes o incomprensibles (p.e.masamas volumen, el carbono, en casi ninguna).

sa-efecto de tipo lineal, $y$ el hecho de que en sus explicaciones tomen en cuenta sólo una o dos variables, de todas las involucradas.

\section{SECCIÓN III}

Todos los años durante la primavera y el verano austral los pingüinos de Magallanes retornan a las playas de Cabo Virgenes, así como a otras zonas costeras de la Patagonia, formando agrupaciones de hasta un millón de organismos. Las variaciones estacionales pueden ser estimadas a partir del cálculo de la densidad de nidos ocupados cada temporada.

Responde poniendo mucha atención:

3.1. ¿Cómo podemos calcular la densidad de nidos?

3.2. ¿Qué factores intervienen para que aumente la densidad de nidos ocupados?
3.3. ¿Cuáles son los factores que determinan un descenso de dicha ocupación?

3.4. ¿En qué caso se produce crecimiento de la población de pingüinos?

\section{Objetivos}

Al emprender el estudio de una población, la densidad suele ser el primer atributo de ésta al que se dedica atención. La densidad se define como la magnitud de la población-número de individuos o biomasa-en relación con alguna unidad de espacio (volumen o superficie). Por este motivo se realiza la pregunta 3.1 , que tiende a averiguar conocimientos procedimentales, nociones asociadas a los métodos de muestreo, así como la habilidad para expresar los resultados utilizando unidades convencionales. Las tres cuestiones restantes $(3.2,3.3$ y 3.4) se analizan de manera integrada por cuanto responden a problemas complementarios asociados al crecimiento poblacional y a la presencia de factores extrínsecos $\mathrm{e}$ intrínsecos que influyen sobre éste.

\section{Comentarios}

Los alumnos hacen referencia preferentemente a la cantidad de nidos presentes o a la cantidad de individuos al proponer los métodos de cálculo, de lo que puede deducirse que el concepto utilizado es el de abundancia, en reemplazo del concepto de densidad de la poblacion (categorías $C$ y D en el gráfico 6). Puede observarse que los profesores en formación alcanzan los valores mayores en la categoría $\mathrm{B}$ seguidos por los alumnos del nivel primario, en tanto que los estudiantes de $3 \mathrm{er}$. curso tienen los porcentajes más elevados de la población en la categoría $A$. Ambas categorías aluden al concepto de densidad de la población con grados distintos de complejidad, En uno de los grupos el alumnado utiliza el concepto físico de densidad, como consecuencia de la enseñanza reciente de éste por parte del docente de la asignatura. Destacamos también el hecho de que un porcentaje elevado de estudiantes no responde esta cuestion (32\% de la población total).

En el gráfico 7 pođemos ver, como en otras cuestiones, las respuestas concentradas en la categoría $\mathrm{C}$, que hace referencia a los factores bióticos y abióticos de manera global sin precisar factores relevantes desde el punto de vista de la dinámica poblacional. Los estudiantes que aluden de manera directa o indirecta a los factores extrínsecos e intrínsecos que afectan a las poblaciones alcanzan un porcentaje en la muestra total del $21 \%$. De nuevo se registran preferentemente respuestas ecologistas (categoría D) en los más pequeños. Sólo el $2 \%$ de la población total contribuye a la categoría $\mathrm{A}$.

\section{SECCIÓN IV (tomado de Baker y Allen 1970).}

Observa atentamente la figura que se presenta a continuación. Cada curva muestra las variaciones en el núme- 
ro de aduitos de una población, en un cierto tiempo.

Explica qué organismo ( $\mathrm{A} \circ \mathrm{B}$ ) es la presa y cuál es el depredador. ¿En que te basas para decirlo?

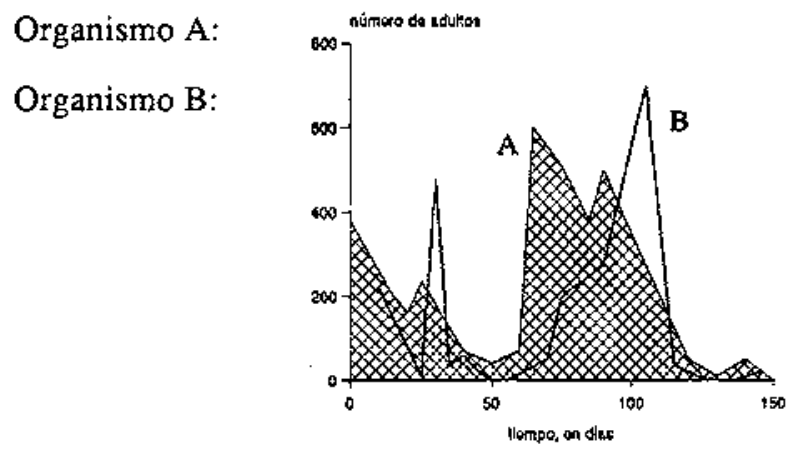

\section{Objetivos}

Las relaciones numéricas entre las poblaciones de depređadores y sus presas son unas de las más llamativas y fructíferas desde el punto de vista educativo, al tiempo que ejemplifican uno de los mecanismos de regulación poblacional. La cuestión planteada tiene como objetivo que los alumnos identifiquen las relaciones entre las variaciones numéricas de la presa y el depredador aportando una respuesta fundamentada; igualmente permite abordar la noción de relaciones interespecíficas y tomar en cuenta la dimensión temporal de estos procesos. Develay y Ginsburger-Vogel (1986) analizaron las explicaciones que algunos alumnos dan a un ejercicio sobre las relaciones entre caribúes, lobos y líquenes en el Canadá. Sostienen que las relaciones alimentarias constituyen una buena aproximación a los problemas de fluctuaciones poblacionales donde los alumnos suelen formular más o menos hábilmente todos los criterios que intervienen (la cantidad de alimento disponible, la concurrencia interespecífica, la tasa de fecundidad). Los resultados obtenidos se presentan en el gráfico 8.

\section{Comentarios}

Los estudiantes de los grupos consultados coinciden en su mayoría en que el organismo A es la presa y B es el depredador. Si bien se interpreta que en general se realiza una lectura correcta del gráfico, algunas de las explicaciones que proporcionan permiten apreciar que algunos de estos alumnos establecen una comparación entre las dimensiones de las curvas y el tamaño real de la presa y del depredador, por cuanto señalan a la curva A como correspondiente al depredador interpretando que el cazador es siempre más grande y oscuro.

La mayoría de los alumnos de 3er. año del ciclo básico no han contestado la pregunta y los que lo han hecho han considerado las dos posibles respuestas en proporciones muy parecidas. Al analizar las explicaciones que dan a la
Gráfico 8

Ideas acerca de las relaciones entre presas y depredadores.

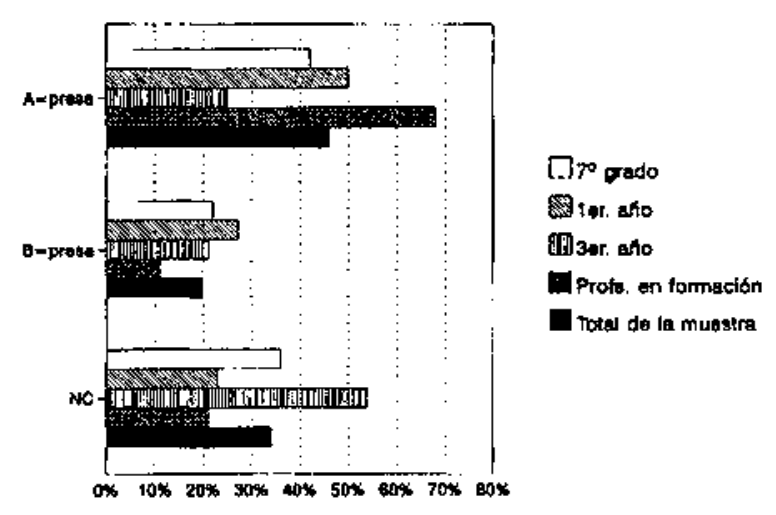

respuesta se puede verificar que, ya se trate de una u otra altemativa, la justificación es idéntica: cuando hay más presas disponibles hay más depredadores y si el número de presas disminuye, el número de depredadores disminuye.

Pozo y otros (1991) explican la manera cómo percibimos las personas las relaciones causales utilizando las reglas de inferencia y la terminología propuestas por Hume. Por lo general, hay una tendencia a atribuir una correspondencia cuantitativa entre causa y efecto: un cambio en la cantidad de efecto debería corresponderse a un cambio en la cantidad de causa y viceversa. Estos autores coinciden en señalar que el razonamiento espontáneo de los alumnos sobre fenómenos científicos se basa en una causalidad lineal simple y suelen recurrir a interpretaciones aditivas más que interactivas.

El diseño del gráfico plantea la relación que se establece de manera clara pero no obvia, siendo necesario interpretar la situación al tiempo que se realiza una lectura matemática de la curva. Ello hace suponer que un tipo de planteamiento simplista que tenga en cuenta más las relaciones aditivas que interactivas conduce a decir que la presa es B sólo por la altura que alcanza la curva, sin tomar en consideración los procesos que se dan en el transcurso del tiempo.

SECCIÓN $V$ (tomado de Del Ponte y Martínez Fontes 1969). Algunos biólogos estudiaron tres poblaciones de «ranas»: A, B y C.

En condiciones naturales:

- La población A se puede cruzar con la B.

- La población $\mathrm{B}$ puede cruzarse con la $\mathrm{C}$.

- Las poblaciones A y C no pueden cruzarse entre sí.

5.1. ¿Cuántas especias de «ranas» están involucradas en este estudio? 
Gráfico 9

Concepciones alternativas sobre el concepto de especie.

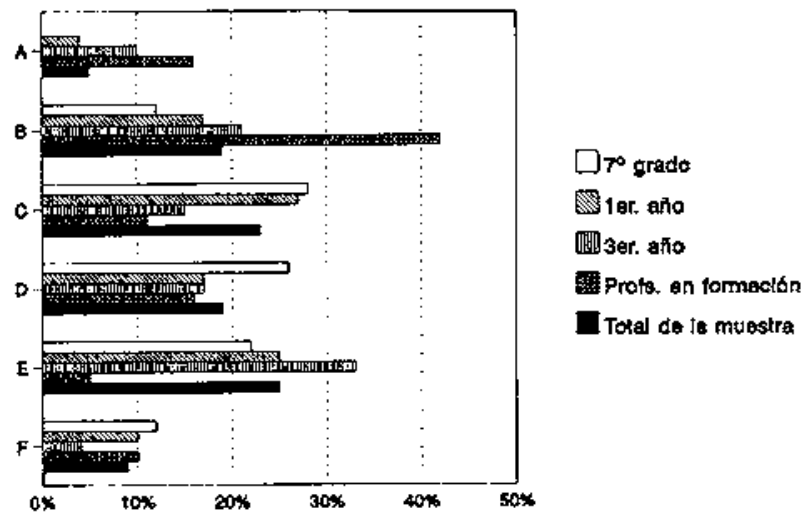

A. Respuestas que consideran el concepto biológico de especie (p.e. $2-2-2,2-2-1,2-1-2,2 \cdot 1-1,1-2 \cdot 1)$.

B. Respuestas que toman en cuenta la reproducción, si bien no distinguen barreras reproductivas (p.e. 5-3-2, 5-2-2).

C. Respuestas que consideran la especie como equivalente a población (p.e. $3 \cdot 2-2,3-2-1$ ).

D. Respuestas que corresponden a un concepto tipologico de especie; la especie es un indiviouo, ambos sexos son de la misma especie o bien constituyen especies distintas (p.e. 2-/-0, /-2-0, 3-2-0).

E. No contestan.

F. Respuestas confusas, incoherentes o incomprensibles (p.e. 4-I, $2-0-1,3-2-3$ y otras).

5.2. ¿Cuántas poblaciones quedarían si $\mathrm{A}$ se extinguiera?

5.3. ¿Cuántas especies habría si desapareciera la población $\mathrm{B}$ ?

Explica el razonamiento que determina tus respuestas.

\section{Objetivos}

Las relaciones existentes entre los conceptos de población y especie son muy importantes. La noción de especie puede constituir un obstáculo para la comprensión del concepto de población y su campo conceptual. La relevancia de este concepto dentro del campo conceptual de población justifica que en el cuestionario se haya incluido un problema específico, a fin de comparar los resultados obtenidos en un contex to predominantemente teórico (Sección I) y en otro contexto más práctico (Sección V).

\section{Comentarios}

Las soluciones propuestas por los atumnos (Gráfico 9) permiten confirmar los resultados obtenidos en relación con el concepto de especie en la Sección I, en cuanto que predomina el concepto «tipológico» (D) en un porcentaje importante de la población. Las explicaciones de los estudiantes destacan en este caso que las poblaciones A y $\mathrm{C}$ son de hembras y la población $\mathrm{B}$ de machos, y los más pequeños llegan incluso a mencionar que cada sexo corresponde a una especie distinta. El alumnado del 3er. curso y los profesores en formación ya han superado esta concepción y asumen que ambos sexos pertenecen a la misma especie. No obstante todos coinciden en reconocer a la especie como un arquetipo. Otro sector del grupo considerado identifica las especies con las poblaciones (categoría C). En las propuestas de solución al problema hay estudiantes que tienen en cuenta la reproducción entre los individuos (categoría B), aun cuando su interpretación es incorrecta, debido a que no reconocen las barreras reproductivas que existen entre las distintas especies. Finalmente hay que constatar que aparece un porcentaje mayor de individuos que los detectados en la Sección I (Gráfico 2), que parecen considerar el concepto «biológico» de especie» (5\%).

\section{DISCUSIÓN Y CONCLUSIONES}

La población en cuanto conjunto de individuos puede «observarse» de manera más o menos directa, por lo que su existencia (como agregado de unidades) es percibida por la totaliđad del alumnado consultado sin mayores dificultades, y sus interpretaciones son coherentes con un pensamiento de tipo concreto, basado en datos recogidos mediante procesos sensoriales y perceptivos. Un análisis comparativo de las respuestas obtenidas permite detectar como rasgo sobresaliente de sus ideas el predominio de las concepciones antropocéntricas en relación con los conceptos de individuo y población. Esta característica puede interpretarse por comparación con los antecedentes históricos de estos conceptos como una similitud entre las ideas de los alumnos y el pensamiento poblacional existente hasta el siglo pasado; el concepto de población nace asociado justamente a la necesidad de comprender y controlar la población humana.

La obligación de extrapolar el concepto de población humana a la población biológica en general requiere superar la visión antropocéntrica $e$ incorporar un nuevo concepto, el de especie, cuya comprensión, como se desprende de los resultados obtenidos, plantea diversas dificultades. De una manera notable, los alumnos asimilan el concepto de especie al de raza de los individuos; en otros casos manifiestan concepciones asociadas con un concepto tipológico de especie, aceptando como sus rasgos distintivos las semejanzas morfológicas entre los organismos que la componen.

La consideración de las relaciones mutuas existentes entre ambos conceptos es reconocida por un porcentaje reducido de la muestra y, aun en el caso que se señale la pertenencia de los individuos de una población a una especie determinada, las ideas acerca de lo que constituye una especie no se corresponden con el concepto aceptado en biología. Puede asumirse entonces que el 
concepto de especie constituye un obstáculo para la comprensión del concepto de población y que el aprendizaje de ambos conceptos debe estar necesariamente relacionado. Para la noción de especie se ha indicado que su acabada comprensión requiere la asimilación de otro concepto, el de interfecundidad. Debe tenerse en cuenta que los estudiantes hacen referencia a la función de reproducción como inherente a los organismos vivos, pero en las preguntas que requieren este tipo de respuesta no manifiestan comprender la necesidad de barreras reproductivas entre las especies ni el requisito de una descendencia fértil para la supervivencia de éstas.

Las referencias al espacio son relativamente amplias en toda la muestra, si bien la percepción que pone de manifiesto el grupo se corresponde con la visión del hábitat como un todo global, lo que constituye una dificultad para apreciar la trama de relaciones que se establecen dentro de él (fenómenos asociados a la đensidad y dispersión de organismos, por ejemplo).

En cuanto a la variable tiempo, es tomada en cuenta sólo en relación con planteamientos y ejemplos muy concretos, y por lo general está ausente de las definiciones propuestas sobre el concepto de población. EI tiempo es, por lo tanto, otro problema que debe destacarse en relación con este concepto y constifuye un obstáculo para la comprensión de procesos asociados a la dinámica de las poblaciones. Los atributos emergentes de este nivel de organización, como son las tasas de natalidad y mortalidad, el crecimiento poblacional, etc., requieren necesariamente de la inclusión del tiempo como una variable esencial. Aunque son capaces de considerarlo en lapsos relativamente breves, el problema fundamental se Ies plantea cuando deben referirse a períodos de tiempo prolongados con los cambios quéstos conllevan en los niveles de individuo, población y especie.

Entre las relaciones causales que implican los procesos poblacionales, puede mencionarse que las relaciones alimentarias del tipo depredador-presa son aparentemente comprendidas por un amplio sector de alumnos. La dificultad les aparece una vez más al hacer referencia a las fluctuaciones de ambas poblaciones (la del depredador y la de la presa) con el tiempo. Las interpretaciones proporcionadas por el alumnado a estas fluctuaciones, así como a las variaciones estacionales que caracterizan determinadas especies, toman en cuenta preferentemente los factores extrínsecos (p.e. el clima), haciendo referencia a los factores intrínsecos en el caso que correspondan a atributos muy cercanos al nivel de organismo más que a características poblacionales de mayor jerarquía.

La acción de la institución escolar no parece tener posibilidades de cambiar estos tipos de planteamientos, sino por el contrario, en algunos casos se podría interpretar que contribuye a su génesis y refuerzo; tal es el caso de las concepciones antropocéntricas que se continúan manifestando aún en profesores en formación.

En cuanto a las probables causas de estas concepciones, si tenemos en cuenta los tres grandes grupos en que Pozo y otros (1991) Ias clasifican, se pueden adscribir a los tres origenes que describen en su trabajo (sensorial, social y analógico), lo que puede considerarse como una de las principales causas de que la instrucción tenga poco éxito en cambiar estas ideas. El origen de muchas de estas concepciones se encuentra en el medio social del alumno, por lo que quizás podrían considerarse principalmente como «inducidas», incluyéndose la escuela como fuente de estas ideas. Esta opinión es coincidente con las conclusiones de Ola Adeniyi (1985) para alumnos nigerianos.

Las perspectivas que se aprecian a partir de las conclusiones de este trabajo llevan a sugerir algunas líneas de acción posibles, en relación con la selección y organización de contenidos de las Ciencias Biológicas en el nivel medio de educación, así como en la didáctica de esta asignatura. Algunas de ellas son:

- Tomar en consideración las ideas de los alumnos sobre el concepto de población y su campo conceptual como punto de partida para la conducción del aprendizaje escolar.

- Hacer explícitas, en el plano de Ia enseñanza, las conexiones y relaciones existentes entre los conceptos mencionados, en particular con el concepto de especie, que es probablemente el más determinado porel pensamiento espontáneo del estudiantado.

- Utilizar como marco de referencia la evolución histórica del concepto (Rabinovich 1980, Develay y GinsburgerVogel 1986, Acot 1990), como orientadora para una adecuada selección y secuenciación de los contenidos.

- Conectar el conocimiento escolar con contextos y situaciones próximas al alumnado, de manera que los problemas que aparecen como característicos de Ias poblaciones sean abordados desde una metodología más próxima a la que genera el saber científico, propiciando la superación paulatina de interpretaciones simplistas y acríticas acerca de la naturaleza y los sistemas ecológi$\cos$.

- Reestructurar los materiales de enseñanza y aprendizaje que se utilizan habitualmente, tomando en consideración las ideas de los alumnos, Ia importancia del concepto de población y su campo conceptual dentro de la teoría ecológica y de la biología en general y las orientaciones que surgen de un estilo de enseñanza basada en el tratamiento de problemas relacionados con el entorno concreto donde interactúan los estudiantes.

\section{Agradecimientos}

M. B. agradece el soporte económico recibido del Instituto de Cooperación Iberoamericana de la Agencia Española de Cooperación Internacional y del Ministerio de Cultura y Educación de Ia provincia de Santa Cruz (Argentina), el cual hizo posible su estancia en la Universitat de València y la realizacíńn de este trabajo. 


\section{REFERENCIAS BIBLIOGRÁFICAS}

ACOT, P., 1990. Historia de la Ecología. (Taurus: Madrid).

BAKER, J.J.W. y ALLEN, S.E., 1970. Biologia e Investigación Científica. (Fondo Educativo Interamericano: México).

BEGON, M., HARPER, J. y TOWNSEND, C., 1988. Ecologia: Individuos, poblaciones y comunidades. (Omega: Barcelona).

DEL PONTE, E. y MARTÍNEZ FONTES, E., 1969. Biología I, adaptación para la región templada de Sudamérica del BSCS, Version Verde. (CONICET/INEC: Buenos Aires).

DEVELAY, M. y GINSBURGER-VOGEL, 1986. Population, Aster, 3, pp. 19-71.

DINEMS-DIEPE, 1978. Guía normativa para la aplicación de nuevas estrategias. (Ministerio de Cultura y Educacion: Buenos Aires).

DINEMS-DIEPE, 1980. Circular núm. 15. (Ministerio de Cultura y Educación: Buenos Aires).

DRIVER, R., GUESNE, E. y TIBERGHIEN, A., 1989. Ideas científicas en la infancia y la adolescencia.(Morata: Madrid)

GONZÁLEZ GARCIA, F.M. 1992. Los mapas conceptuales de J.D. Novak como instrumento de investigacion en la didáctica de las ciencias experimentales, Enseñanza de las Ciencias, $10(2)$, pp. 148-158.
HAWLEY, A., 1975. Ecología Humana. (Tecnos: Madrid).

ODUM, E., 1972. Ecología. (Interamericana: México).

OLA ADENIYI, 1985. Misconceptions of selected ecological concepts held by some Nigerian students, Journal of Biological Education, 19(4), pp. 311-316.

POZO, J.I., SANZ, A., GOMEZ CRESPO, M.A. y LIMÓN, M., 1991. Las ideas de los alumnos, sobre la ciencia: una interpretación desde la psicología cognitiva, Enseñanza de las Ciencias, 9(1), pp. 83-94.

RABINOVICH, J., 1980. Introducción a la ecología de poblaciones animales. (Continental: México).

SERRANO, T. y BLANCO, A., 1988. Las ideas de los alumnos en el aprendizaje de las ciencias. (Narcea: Madrid).

SNEATH,P.H.A.y SOKAL, R.R., 1973. Numerical Taxonomy. The Principles and Practice of Numerical Classification (W.H. Freeman \& Co.: San Francisco).

TREAGUST, D.F., 1988. Development and use of diagnostic test to evaluate student's misconceptions in Science, International Journal of Science Education, 10(2), pp. 88-96.

YUSRAMOS,R., 1989. El aprendizaje de la ecología, Cuadernos de Pedagogía, 175, pp. 42-45. 